\title{
Experiment for Determination of Residual Stresses in Pipes
}

\section{Shimov Georgy}

Ural Federal University named after the First President of Russia B. N. Yeltsin, Ekaterinburg, Russia

\section{Abstract}

The paper describes the method of the metal surface layer etching from the pipes and the measurement of elastic relaxation for the analysis of the residual stresses of cold-rolled stainless steel pipes. The results of the experimental determination of the residual stresses of pipes made of steel AISI 321 are given.

Keywords: residual stresses, measurement of residual stresses, stresses distribution, tubes for nuclear steam generators, etching, experimental installation

Corresponding Author:

Shimov Georgy

g.v.shimov@urfu.ru

Received: 25 February 2019

Accepted: 9 April 2019

Published: 15 April 2019

Publishing services provided by

Knowledge E

(c) Shimov Georgy. This article is distributed under the terms of the Creative Commons

Attribution License, which permits unrestricted use and redistribution provided that the original author and source are credited.

Selection and Peer-review under the responsibility of The Ural school-seminar of metal scientists-young researchers Conference Committee.

\section{G OPEN ACCESS}

\section{Introduction}

Practically all methods of non-destructive testing of residual stresses in metal products, such as X-ray, ultrasonic, optical, magnetic, etc., used for the residual stresses measuring between the atoms of the metal crystal lattice [1-3]. At the same time, residual stresses of the second kind (intergranular and subgrain stresses), and third kind (macrostresses, balanced in the volume of the whole body) practically cannot be captured by means of nondestructive testing [4]. The most common method among the methods of destructive control of residual stresses are the methods of mechanical dismemberment, for example, the methods of drilling, cutting (bands or rings) and layer-by-layer cutting [10]. The essence of these methods is the indirect determination of residual stresses based on the results of the capturing of the body elastic response to an external mechanical effect.

To capture the residual stresses of the third kind in the pipes, the method of cutting rings is used. The essence of the method consists of cutting off a thin ring from the pipe, cutting it by the generatrix and then the elastic compression or ring opening capturing at the cut point. This method determines the tangential residual stresses in the pipe. The disadvantages of this method are obvious and known. The fact is that at the time of the ring cutting off, the field of residual stresses inevitably changes (including tangential ones) and actually residual stresses in a single ring are measured, which may not correspond to a stresses in the original pipe [9]. 
The above-mentioned disadvantages are absent in the proposed method of etching. The method consists of a thin metal surface layer removing by electrolytic etching and subsequent capturing of the elastic changes in the geometric dimensions of the body. The method can be applied both in the proposed format of complete etching of the surface layer from the pipe, and in the local etching of some area of the body and capturing of the elastic reactions using strain gauges, similarly to the drilling method. Strangely enough, in the foreign literature there is no description of any etching attempts to assess the residual stresses in metal products. In practice of testing metals, etching is present only as a method of the surface cleaning before using some non-destructive testing methods, and also as a tool for qualitative assessment of the presence or absence of residual stresses in such techniques as, methods of intergranular corrosion testing, for example. At the same time, the correct application of the etching method can ensure a high accuracy of the residual stresses determination, and also a high speed of the tests. Removal of the surface layer of the metal by etching does not lead to a local temperature jumps, does not cause the effects of local vibration or deformation, which ensures the accuracy of the metal elastic reactions capturing.

\section{Design of Installation}

In the developed method of the residual stresses determination, the etching method is used to measure the longitudinal residual stresses in the surface layers of pipes. Pipes for the nuclear power industry (the experiments of such pipes were performed) were chosen as the object of study for several reasons. First, the pipe is an ideal geometrical object for the elastic unloading's and residual stresses of the third kind capturing. Secondly, the pipes for nuclear power plants (in this case, pipes made of steel AISI 321 with dimensions of $16 \times 1.5 \mathrm{~mm}$ ) are almost perfectly straight, which guarantees the measuring accuracy of the linear elongation. Thirdly, despite of the requirements for residual stresses are not spelled out in almost any standard, this problem has recently been paid attention to. The fact is that when operating in an aggressive environment, tensile residual stresses on the surface of pipes lead to the rapid opening of corrosion ulcers and cracks and the failure of pipe fittings due to a corrosion cracking [6-8].

The work [9] describes a calculation method of the residual stresses determination based on the energy approach [11], which, like the method of cutting rings, is based on the method of splitting, but involves the method of the pipe surface layer etching. After the surface layer removing and the elastic relaxation along the length capturing, the residual stresses are calculated over the entire cross section of the pipe wall. 
For the practical implementation and testing of the methodology proposed in [10], it is necessary to develop the design of the installation for a pipe thin surface layer etching. An installation intended for carrying out of the experiments on longitudinal residual stresses measuring should ensure a high uniformity of the surface layer etching, have a convenient procedure for pouring and draining electrolyte, and should also ensure quick and convenient sample placing and removal. In addition, the installation must ensure work safety for the operator and others, therefore, the design must be stable in order to prevent tipping of the electrolyte bath over. One of the method implementation problems is that during the etching process reaction products are not evenly removed from the surface. In the upper part, the formed bubbles float freely in the electrolyte, and in the lower part of the sample a layer of bubbles "sticking" forms, which disrupts the normal kinetics of the process.

During the installation design for solving the above problems a continuous sample rotation was used during the etching process in the electrolyte bath. The complexity of the design solutions for torque supplying to the sample is determined by the aggressiveness of the etching environment, as well as the need for a continuous current supply to the sample. The only possible design option for the installation is to apply torque to the sample through a flexible drive rotation, and the current can be supplied through a rolling bearing mounted directly to the sample.

\section{Measurement Technique}

The technique of the residual stresses measuring is as follows:

1. Measurement of the initial dimensions of the sample: initial sample length $L_{0}$, initial outer diameter $D_{0}$, initial wall thickness $S_{0}$;

2. Etching of a thin surface layer from the outer or inner surface of the sample;

3. Measurement of the final dimensions of the sample: the final length of the sample $L_{1}$, the final outer diameter $D_{1}$, the final wall thickness $S_{1}$;

After the measurements the distribution of residual stresses of the pipe wall or in the bar section is calculated by a specially developed calculation method [10]

The measuring stand of the sample length is a rigid metal frame made of a thick metal profile with a fixed and movable cross-bar. A measuring head is mounted in the movable cross-bar. The appearance of the measuring stand is shown in Fig. 1.

Before the start of the experiment the initial sample length is changed. Sample is installed between the holders, mounted at the fixed 8 and movable 3 cross-bar of the 


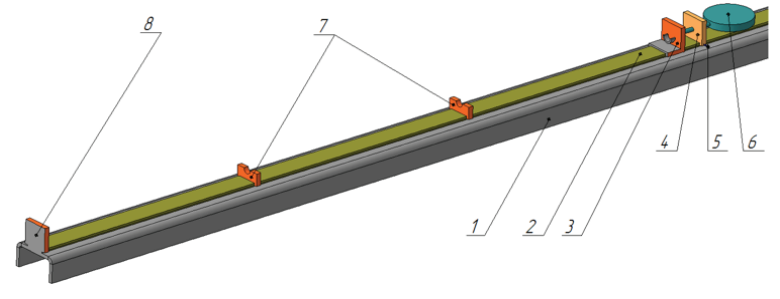

$a$

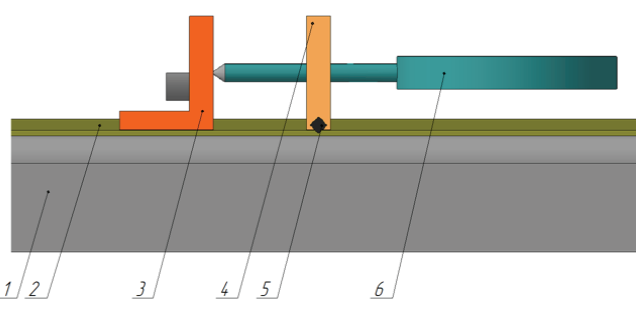

$b$

Figure 1: Appearance of the measuring stand: $a-3 D$-view of the stand; $b$ - side view of the measurement unit: 1 - rigid frame; 2 - guide bar; 3 - mobile cross-bar; 4 - mobile control rack; 5 - locking screw control rack; 6 - indicator; 7 - supporting intermediate racks; 8 - fixed cross-bar.

stand. The movable cross-bar 3 along the guide bar 2 is clamped up to the sample. After that, the movable control rack with the indicator is fed to the movable cross-bar and fixed with screw 5 . Control rack must be fixed in a position where indicator 6 shows a value of $0.5 \ldots 1.0 \mathrm{~mm}$, in which case the indicator can capture a sample elongation after etching and its shortening.

For the accuracy improvement length measurements should be carried out in a several positions of the sample with rotation through the angle $a=45^{\circ}$ ( 4 positions in total), then it is possible to calculate the average value of the absolute change in the sample length

$$
\Delta L_{c p}=\frac{\sum_{i=1}^{n}\left(L_{c p 0 i}-L_{c p 1 i}\right)}{n}
$$

where $L_{c p 0 i}$ - the length of the sample before etching in position $i, L_{c p 1 i}$ - the length of the sample after etching in position $i, n$ - the number of length measuring positions of the sample $(n=4)$ (Fig. 2, a). This can help to exclude the total sample curvature possible impact on the measurement accuracy, as well as its local deflection when placed on the measurement stand. Risks with numbers of sections are applied by the blade on the surface of the end caps, so that they will not be erased during the etching process (Fig. 2, b).

An important aspect of the measurement technique is the preparation of sample pipes or bars for the experiment. The sample length should not be too short in order to improve the accuracy of the sample elastic deformation measuring after etching. It was experimentally established that the optimal length of the sample for the surface layer etching with a thickness of $0.05 \ldots 0.08 \mathrm{~mm}$ can be $L=1500 \mathrm{~mm}$. The ends of the specimens must be reliably insulated to prevent acid from entering them. Also working length of the etching surface should be clearly highlighted. In the case of the outer surface layer etching the sample is insulated in accordance to a Fig. 3, a. In this case additional end insulation is carried out using liquid molten kapron or varnish, as well as the separation of the length of the etching surface on the outer surface. In the case of the 


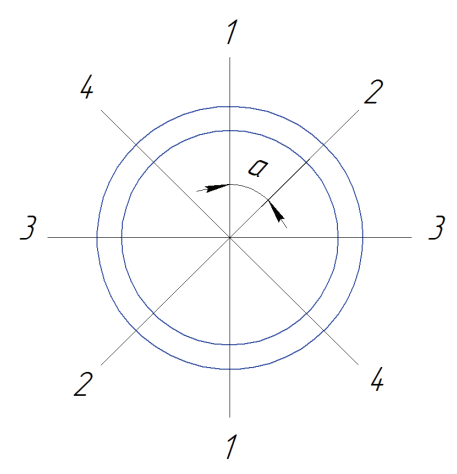

$a$

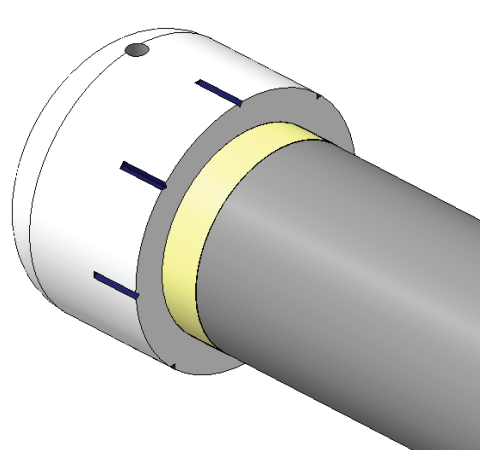

$b$

Figure 2: The position of the sample in which diameter and length measurements are made before and after metal layer etching: $a$ - number (i) of the sections; $b$ - marks on the end cap of the sample.

inner surface layer etching the insulation of the pipe sample is carried out in accordance to a Fig. 3, b. With such an isolation scheme the electrolyte should freely fall inside the sample and the entire outer surface should be insulated in order to prevent acid from getting into it during the experiment.

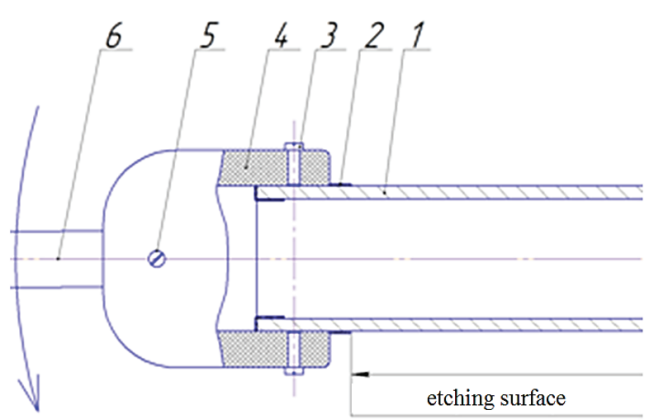

$a$

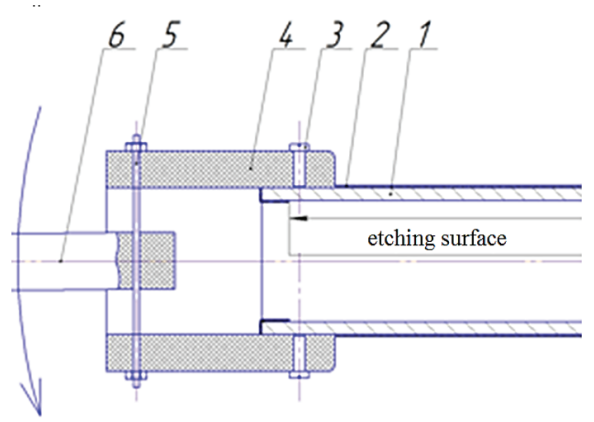

$b$

Figure 3: The scheme of the sample end insulation during the outer surface layer etching $(a),(b)$ - inner: 1 sample; 2 - insulation (varnish / kapron); 3 - clamping screw; 4 - end cap; 5 - pinprick; 6 - drive shaft.

The process of sample preparation is carried out in the following sequence:

1. Measured length sample cutting off from pipe or bar;

2. Bearing fastening in the center of the sample;

3. Kapron layer or varnish applying to the outer / inner surface in order to insulate the ends of the sample and establish a fixed length of the etching surface;

4. Insulation of the outer surface of the sample (in the case of the inner surface etching) using kapron or varnish;

5. End caps installation;

6. End caps with clamping screws fastening; 
7. Drive shafts with pinpricks fastening.

Insulation of the ends is the most convenient to carry out by lowering the sample to a container with a prepared insulating liquid (kapron / varnish) in a vertical position. In this case it is easy to ensure the accuracy of the insulation applied by controlling the level of the insulator in the tank.

Insulation of the outer surface can be done in two ways. The first method is to apply a thin layer of insulator (kapron or varnish) by analogy with the insulation of the ends of the sample. However, this method is complicated and does not guarantee one hundred percent insulation. The second way is to install an external protective cover on the principle of "pipe in pipe". As such a cover a polypropylene pipe can be used the inner diameter of which is slightly larger than the outer diameter of the sample. When implementing the second method of the outer surface insulating, it is necessary to prevent electrolyte from entering to the gap between the sample and the protective cover. After preparation of the sample, it together with the fixed flexible shafts, is lowered into the electrolyte and fixed in the holders of the installation. Then electric connections from the current source are attached to the bearing and bath, the bath is filled with the electrolyte until the sample is completely immersed and one of the flexible shaft is mounted in the clamps of the rotating motor. In this state the installation is ready for operating (Fig. 4). The used electrolyte was an acid solution containing $\mathrm{H}_{3} \mathrm{PO}_{4}-86 \%, \mathrm{Cr}_{2} \mathrm{O}_{3}-12 \%$ (in grams) and $\mathrm{H}_{2} \mathrm{SO}_{4}-2 \%$. In the experiments an electric current rectifier was used, which allowed regulating both the output current and the voltage during operation.

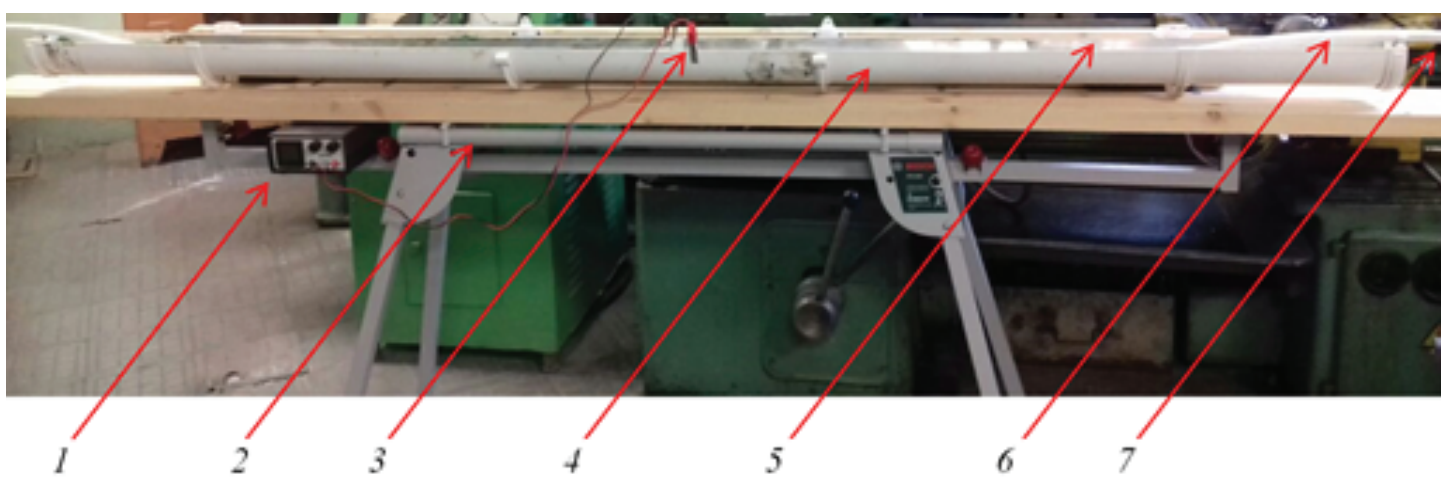

Figure 4: Bath: 1 - current source; 2 - bed; 3 - connections; 4 - bath; 5 - steel tape; 6 - flexible drive; 7 - shaft leading to the engine.

The installation operation begins with the inclusion of a rotation drive. It is necessary to ensure that the sample rotates smoothly with respect to the axis of the installation, there are no strong beats, no leaks or splashes of electrolyte. If all the structural elements work correctly, clamps with wires leading to the current source are installed on the bearing and bath, etching time is detected and voltage is applied. Before switching on 
the current source, the required values of voltage and current are set on it. After starting the installation an electrochemical etching reaction begins to take place over the entire surface of the sample, which can be visible: the formation of reaction products, gas formation and bubbling occur along the entire length of the sample.

After the end of the estimated etching time, the current source is switched off, the connections with wires are disconnected from the bearing and bath, the motor is disconnected from the flexible rotation drive and the sample and the flexible drive are removed from the installation. Then it is necessary to wash the sample and dry it. After drying the flexible drives are disconnected from the sample, the end caps, the bearing and the insulator layer are removed from the ends of the sample. After etching the sample is similarly placed on the measuring stand, its length is measured and the absolute elongation or shortening is calculated using formula (1).

\section{Experiment and Discussion of Results}

At the first stage of the experiment it was necessary to establish the thickness of the etched layer, sufficient for fixing the elastic unloading along the length $\Delta L$. Etching was carried out from the outer surface of two samples to a depth of $0.03 \mathrm{~mm}$ and $0.06 \mathrm{~mm}$, respectively. The absolute change in the length of the samples measured by the method described above, was $\Delta L_{c p 1}=0.04 \mathrm{~mm}$ and $\Delta L_{c p 2}=0.11 \mathrm{~mm}$, respectively. Thus, it was decided that the thickness of the etched layer should be about $0.06 \mathrm{~mm}$. A positive value (absolute elongation) of the sample is explained by the presence of tensile residual stresses on the outer surface of the sample. Metal residual stresses are naturally reactive and are the elastic reaction of the metal to the "resistive load". Therefore, the removal of a thin surface layer of metal with tensile residual stresses is equivalent to the removal of some compressive external load from the sample. That is, if there are compressive residual stresses on the surface of the sample, then the sample is shortened after etching and $\Delta L_{c p}>0$, and vice versa, if tensile residual stresses are present on the surface of the sample, the sample after etching is extended and $\Delta L_{c p}<0$.

In the main experiment 18 samples were cut from pipes of $16 \times 1.5 \mathrm{~mm}$ made of steel AISI 321. 11 samples were prepared for the outer surface etching, 7 - for the inner surface etching (Fig. 5). The etching modes, as well as the measurement results of the samples relaxation are given in Table. $\mathbf{1}$.

After the results analyzing for the sharply thrown values (by elimination of gross errors by the maximum relative deviation) samples No.5 and No.16 were excluded from the analysis. Sample number 13 (short) is passed the analysis. Thus good repeatability of 


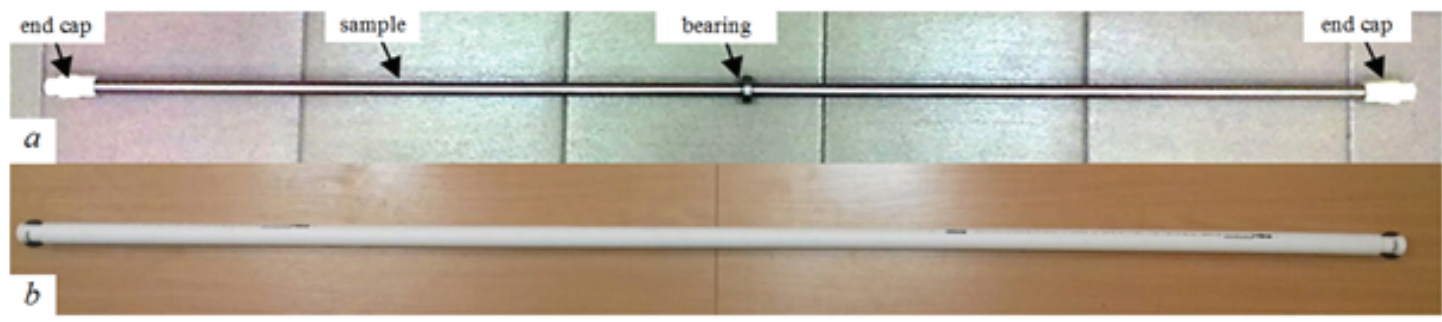

Figure 5: Samples for the experiment: $a$ - for the outer surface etching, $b$ - for the inner surface etching.

TABLE 1: Etching modes and relaxation measurement results.

\begin{tabular}{|c|c|c|c|c|c|c|c|c|c|c|}
\hline № & $L_{c p 0}, \mathrm{~mm}$ & $d_{0}, \mathrm{~mm}$ & $t, \min$ & $L_{c p 1}, \mathrm{~mm}$ & $d_{1}, \mathrm{~mm}$ & $\Delta \mathrm{L}_{c p}, \mathrm{~mm}$ & I, A & Note & $\sigma_{z z}, \mathrm{MPa}$ & $\sigma_{\varphi \varphi}, \mathrm{MPa}$ \\
\hline \multicolumn{11}{|c|}{ Oter surface etching } \\
\hline 1 & 1480,10 & 16,011 & 100 & 1480,19 & 15,881 & 0,09 & 2 & & 119,37 & 397,91 \\
\hline 2 & 1482,30 & 16,008 & 100 & 1482,38 & 15,878 & 0,08 & 2 & & 105,73 & 352,44 \\
\hline 3 & 1483,80 & 16,007 & 100 & 1483,91 & 15,824 & 0,11 & 2 & & 108,56 & 361,85 \\
\hline 4 & 1480,60 & 16,012 & 120 & 1480,74 & 15,805 & 0,14 & 2 & & 117,67 & 392,22 \\
\hline 5 & 1481,60 & 15,995 & 120 & 1481,81 & 15,787 & $\underline{0,24}$ & 2 & excl.. & 130,11 & 433,71 \\
\hline 6 & 1480,40 & 16,011 & 120 & 1480,52 & 15,855 & 0,12 & 2 & & 118,95 & 396,50 \\
\hline 7 & 1480,90 & 16,022 & 90 & 1480,98 & 15,905 & 0,08 & 2 & & 103,32 & 344,41 \\
\hline 8 & 1479,50 & 16,000 & 90 & 1479,57 & 15,883 & 0,07 & 2 & & 122,26 & 407,52 \\
\hline 9 & 1480,50 & 16,050 & 90 & 1480,620 & 15,927 & 0,12 & 2 & & 135,79 & 452,63 \\
\hline 10 & 1481,50 & 15,990 & 180 & 1481,730 & 15,730 & 0,23 & 2 & & 114,44 & 381,47 \\
\hline \multirow[t]{2}{*}{11} & 1480,50 & 16,015 & 180 & 1480,735 & 15,755 & 0,19 & 2 & & 119,37 & 397,91 \\
\hline & \multicolumn{10}{|c|}{ Inner surface etching } \\
\hline 12 & 1476,00 & 14,520 & 100 & 1475,87 & 14,650 & 0,13 & 2 & & $-119,35$ & $-397,84$ \\
\hline $13 \kappa$ & 1464,00 & 14,500 & 100 & 1463,86 & 14,630 & 0,14 & 2 & short. & $-131,68$ & $-438,94$ \\
\hline 14 & 1475,00 & 14,525 & 100 & 1474,90 & 14,681 & 0,10 & 2 & & $-117,84$ & $-392,80$ \\
\hline 15 & 1476,00 & 14,450 & 90 & 1475,92 & 14,580 & 0,08 & 2 & & $-121,97$ & $-406,56$ \\
\hline 16 & 1477,00 & 14,500 & 90 & 1476,99 & 14,617 & $\underline{0,01}$ & 2 & excl.. & $-134,28$ & $-437,18$ \\
\hline 17 & 1465,50 & 14,480 & 180 & 1465,33 & 14,714 & 0,17 & 2 & & $-125,93$ & $-419,76$ \\
\hline 18 & 1478,50 & 14,520 & 180 & 1478,33 & 14,754 & 0,17 & 2 & & $-119,35$ & $-397,84$ \\
\hline
\end{tabular}

the measurements results of the absolute length change of the samples was revealed, which may indicate to a correct sample preparation and the correctness of the length measurement method. After measuring the elastic relaxation of the samples, we calculated the longitudinal residual stresses on the surface of the pipes and the distribution of the residual stresses in the wall $[10,12]$. The results of the calculations of residual stresses are given in Table. 1. On the graphs of Fig. 6, for example, the distributions of longitudinal, tangential and radial residual stresses over the pipe wall thickness for sample No.1 are given. 

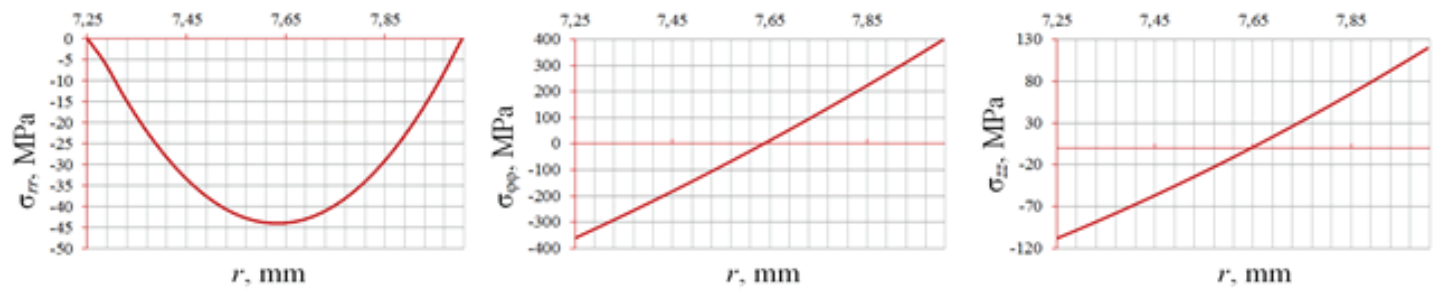

Figure 6: Distribution of the residual stresses across the wall thickness of the pipe (sample No. 1).

\section{Summary}

As a result of the measurements analysis, it can be concluded that there are significant tensile residual stresses on the surface of the pipes, which is due to the technology of their production. For all samples except samples No. 5 and No. 16 the longitudinal residual stresses on the outer surface are within the range of 100 to $155 \mathrm{MPa}$, and on the inner surface of 90 to $135 \mathrm{MPa}$. Tangential residual stresses on the outer surface are in the range of 340 to $495 \mathrm{MPa}$, and on the inner surface - of 315 to $440 \mathrm{MPa}$. Taking into account the fact that procedure is very sensitive to the accuracy of the length measuring of the pipe, the ranges of the resulting stresses are not too large. Thus, we can conclude the correctness of the application of the new method and the calculation methodology for the residual stresses determination.

\section{Acknowledgments}

The study was made within the grant of Russian Science Foundation (№ 17-79-100072)

\section{References}

[1] Schajer, G. S. (2013). Practical residual stress measurement methods. Practical residual stress measurement methods (pp. 1-310) doi:10.1002/9781118402832

[2] Withers, P. J., \& Bhadeshia, H. K. D. H. (2001). Residual stress part 1 measurement techniques. Materials Science and Technology, 17(4), 355-365. doi:10.1179/026708301101509980

[3] Withers, P. J., \& Bhadeshia, H. K. D. H. (2001). Residual stress part 2 - nature and origins. Materials Science and Technology, 17(4), 366-375. doi:10.1179/026708301101510087

[4] Sokolov I.A. Residual stresses and quality of metal products / I.A. Sokolov, V.I. Uralskii. - M.: Metallurgy, 1981. - 96 p. 
[5] Da Silva, L. L., Ribeiro, V. S., Scaldaferri, D. H. B., Rabello, E. G., Campos, W. R. C., \& Mansur, T. R. (2016). Development and test of an equipment to measure residual strain inside pipes using the hole-drilling method. Latin American Journal of Solids and Structures, 13(13), 2439-2450. doi:10.1590/1679-78253126

[6] De Abreu Mendonça Schvartzman, M. M., Quinan, M. A. D., da Costa Campos, W. R., \& Lima, L. I. L. (2011). Stress corrosion cracking of the aisi 316I stainless steel haz in a pwr nuclear reactor environment. Welding International, 25(1), 15-23. doi:10.1080/09507110903569073.

[7] Hu, J., Chen, B., Smith, D., Flewitt, P., \& Cocks, A. C. F. (2013). A self-consistent model in the local residual stress evaluation of $316 \mathrm{H}$ stainless steel. Paper presented at the 13th International Conference on Fracture 2013, ICF 2013, 4, 3351-3360.

[8] James, Malcolm \& Hattingh, D.G. \& Asquith, David \& Newby, Mark \& Doubell, Philip. (2016). Applications of Residual Stress in Combatting Fatigue and Fracture. Procedia Structural Integrity. 2. 11-25. 10.1016/j.prostr.2016.06.003.

[9] M.A. Rozenbaum, A.V. Serebryakov, I. Bazhukov, G.V. Shimov. Study of Residual Stresses and Surface Hardening in Tubes for Nuclear Steam Generators. Solid State Phenomena. 2018. Vol. 265, pp. 53-59. doi:10.4028/www.scientific.net/SSP.265.53.

[10] Shimov G.V. Rapid Method for Determining the Distribution of Residual Patterns in Pipes. Materials Performance and Characterization. Vol. 4, No. 7, 2018. doi: 10.1520 / MPC20170050

[11] Kolmogorov G.L., Kuznetsova E.V., Tiunov V.V. Technological residual stresses and their impact on the durability and reliability of metal products. Perm: Publishing House Perm National Research Polytechnic University, 2012. - 226 p.

[12] Shimov, G., Rozenbaum, M., Serebryakov, A., \& Serebryakov, A. (2016). The method for measuring residual stress in stainless steel pipes. Journal of Chemical Technology and Metallurgy, 51(4), 458-464. 